\title{
Clock-Modulation Based Watermark for Protection of Embedded Processors
}

\author{
Jedrzej Kufel*, Peter Wilson*, Stephen Hill ${ }^{\dagger}$, Bashir M. Al-Hashimi*, Paul N. Whatmough ${ }^{\ddagger}$ and James Myers ${ }^{\ddagger}$ \\ ${ }^{*}$ School of Electronics and Computer Science, University of Southampton, Southampton, UK \\ $\{$ jjk1g10, prw, bmah\}@ecs.soton.ac.uk \\ †ARM Ltd, Austin, US, Stephen.Hill@arm.com \\ $\ddagger$ ARM Ltd, Cambridge, UK, \{Paul.Whatmough, James.Myers\}@arm.com
}

\begin{abstract}
This paper presents a novel watermark generation technique for the protection of embedded processors. In previous work, a load circuit is used to generate detectable watermark patterns in the ASIC power supply. This approach leads to hardware area overheads. We propose removing the dedicated load circuit entirely, instead to compensate the reduced power consumption the watermark power pattern is emulated by reusing existing clock gated sequential logic as a zero-overhead load circuit and modulating the clock-gating enable signal with the watermark sequence. The proposed technique has been validated through experiments using two ASICs in 65nm CMOS, one with an ARM Cortex-M0 microcontroller and one with a Cortex-A5 microprocessor. Silicon measurement results verify the viability of the technique for embedded processors. Furthermore, the proposed clock modulation technique demonstrates a significant area reduction, without compromising the detection performance. In our experiments an area overhead reduction of $98 \%$ was achieved. Through reuse of existing logic and reduction of watermark hardware implementation costs, the proposed clock modulation technique offers an improved robustness against removal attacks.

Index Terms-Watermarking, Embedded Systems, CPA
\end{abstract}

\section{INTRODUCTION}

Technology scaling and innovations in modern processes are allowing increasingly complex systems to be implemented on a single die [1]. To support this design complexity it is increasingly desirable to source sub-systems, such as Central Processing Units (CPU), from external Intellectual Property (IP) suppliers. IP blocks are usually delivered as either hardmacros, full circuit layouts, or soft-macros, typically registertransfer level (RTL) descriptions. The Virtual Socket Interface (VSI) Alliance [2] proposes three approaches to the problem of securing an IP: deterrent, protection and detection. The deterrent approach may deter the infringement from occurring through patents, copyrights, trade secrets, contracts or lawsuits [2]. However, it does not provide physical protection. The protection approach prevents unauthorized use of IP through encryption. Nonetheless, encryption and rights managements support in EDA tools is far from universal and pain-free [3]. Therefore, IP blocks are often supplied as unprotected design files that System-on-Chip (SoC) integrators can use without any complication of their design flow. As a result, auditing the presence of IP in finished products is an important challenge for IP providers. De-encapsulation and die-level

978-3-9815370-2-4/DATE14/(C)2014 EDAA reverse engineering can be used to prove the presence of IP, but the process is slow and costly [3], [4]. It is therefore desirable to identify and prioritize IP candidates to be short-listed for more thorough investigation.

The VSI Alliance proposes digital watermarking as one of detection methods for physical IP protection, at various design flow levels [2]. The use of the soft IP is more desirable as it offers the end user the highest level of flexibility [2]. Methods for the protection of soft IP can be divided into two groups, where architecture of the watermark circuit is closely related to the detection technique.

Watermark detection at output ports of a device through application of input vectors has been reported as one of the IP protection techniques in previous work [5]-[9]. Through an addition of extra states into Finite State Machines (FSM) [5][7], or modification of existing states [8], [9], the area overhead of watermark implementation is reduced, with a reported $0 \%$ in [9]. Nevertheless, the IP vendor may not have sufficient knowledge about the final design to able to use such detection techniques and prove the IP infringement.

In [10], Becker et al applied Correlation Power Analysis (CPA) [11], as a watermark detection technique. The CPA detects an embedded power watermark in the dynamic or static current variations on the supply voltage rail. Therefore, no additional dedicated connections to the input or output ports of a device are required. However, a power watermark requires two circuits: a watermark generation circuit (WGC) and a load circuit (LC) [10], [12]. The architecture of the WGC depends on the watermark sequence. Nonetheless, it is kept relatively small, and 32 registers have been reported in [10], [12]. The LC consists of shift registers and determines the power consumed by the watermark circuit. Its size is closely related to the system size. Zeiner et al report 92 out of 1332 lookup tables (LUT) on FPGA, a $6.9 \%$ of system area, were used with each LUT configured as 16-bit shift register, for simple arithmetic coder core [12]. Similarly, Becker et al utilize 16 LUTs on the Advanced Encryption Standard (AES) cryptographic core [10]. It can be seen, that the majority of area overhead in the current state-of-the-art power watermark architecture is caused by the significant size LC.

Embedded processors are increasingly constrained in terms 




(a)

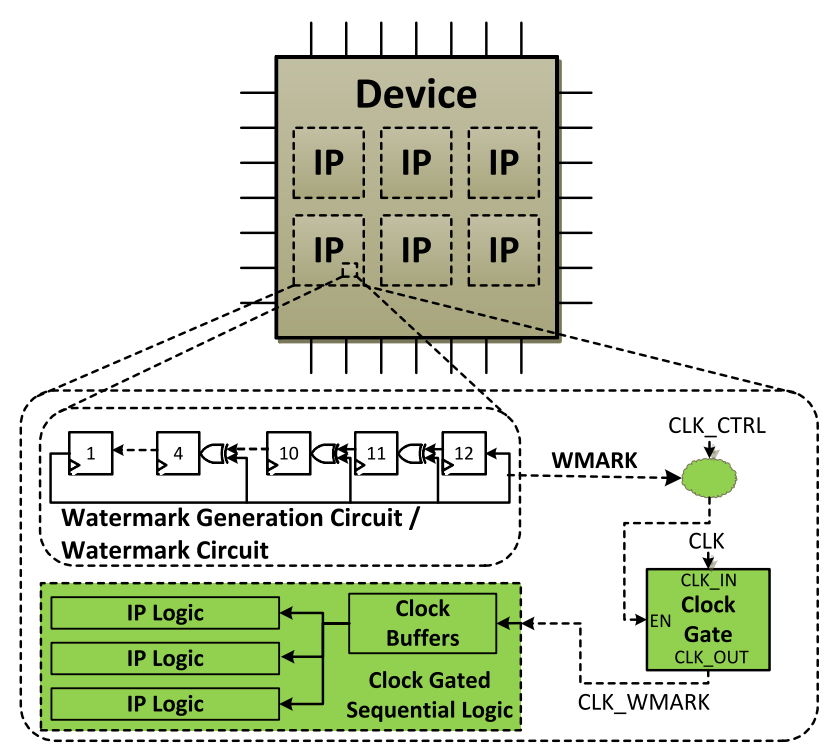

(b)

Fig. 1. Architecture of (a) current state-of-the-art, (b) proposed clock modulation power watermark circuit.

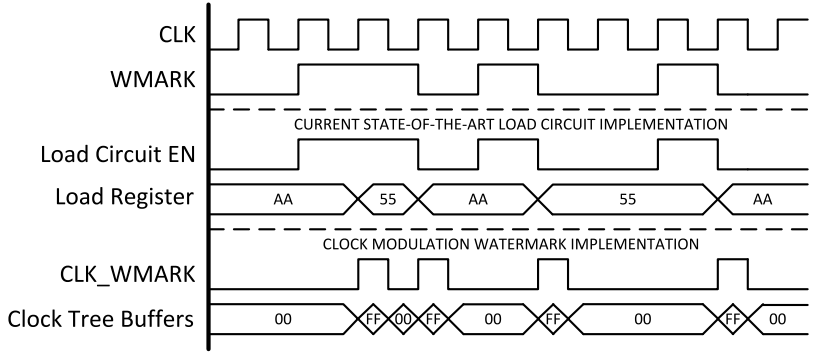

Fig. 2. Timing diagram of current state-of-the-art (middle) and clock modulation (bottom) watermark architectures.

of circuit area. Moreover, a watermark circuit embedded in the soft IP design level is the most vulnerable to third party attacks due to high visibility of the RTL description. It is therefore crucial for the power watermark circuit to have negligible impact on area overhead. The primary motivation of this work is therefore the minimization of power watermark hardware implementation costs, necessary for highly constrained embedded processors. In this work, a novel technique is proposed to generate particular power pattern through the modulation of clock signal, without compromising watermark detectability.

The paper is organized as follows. In Section II, the current state-of-the-art and proposed clock modulation architecture of the watermark circuit are presented. The Correlation Power Analysis watermark detection technique is discussed in Section III. Silicon measurements are given in Section IV. Section V, discusses hardware implementation overheads. The improved robustness against removal attacks of the proposed clock modulation watermark architecture is shown in Section VI. Section VII, concludes the paper.

\section{Clock Modulation Watermark Architecture}

A power watermark is a redundant circuit added to an existing IP block, with the aim of superimposing a weak but deterministic signal on a supply voltage rail. The current stateof-the-art power watermark [10], [12] implements a watermark generation circuit (WGC) and a load circuit (LC), Fig. 1(a). The WGC generates the watermark sequence, WMARK, which controls the shift enable input of the LC, as shown in Fig. 1(a). In Fig. 2, the timing diagram of the watermark circuit is shown. The load register consists of an 8-bit shift register initialized with $1010 \ldots$ pattern, to maximize dynamic power consumption when $W M A R K$ is ' 1 '. It can be seen, that when $W M A R K$ is ' 1 ' the shift enable signal is ' 1 ' and dynamic power is consumed, due to shift operation during which all registers change their states. Analytical techniques such as Correlation Power Analysis (Section III), have been reported to detect deeply embedded watermark signals [10]. However, area overhead of the watermark circuit in Fig. 1(a) is significant, when compared to area overhead as low as $0 \%$ with techniques [5]-[7] discussed in Section I.

To reduce area overhead, we propose a technique which drives an IP sub-module with the watermark modulated clock signal, as shown in Fig. 1(b). In digital circuits, the distribution of a clock signal on a chip, also known as a clock tree [13], contributes a significant amount of dynamic power consumption. In [14], the authors report that typically up to $50 \%$ of the total dynamic power is consumed by the system's clock signal. Since most of the processor design is sequential, with a large number of registers being clocked, the fan-out of the clock tree causes high dynamic power consumption. The technique known as clock gating reduces the dynamic power consumption, with a special clock gating logic cell added to the clock tree, to switch off the parts of the design 
Embedded System Power
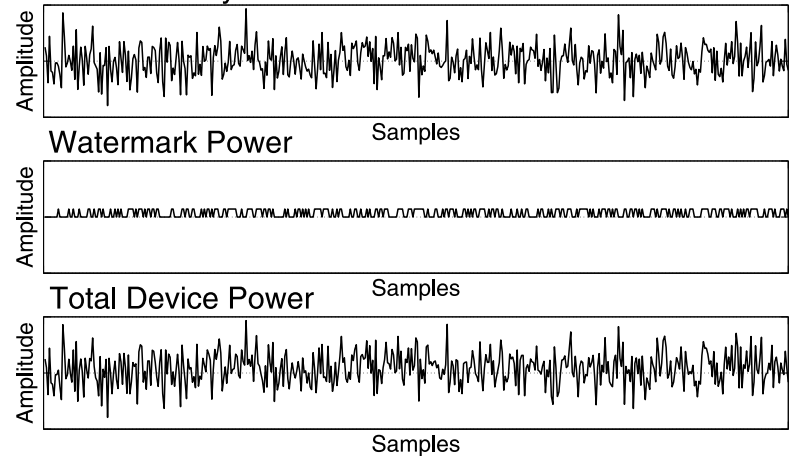

Fig. 3. Simulation results of effect of the watermark power signal on the total device power.

when it did not need to be updated [15]. To utilize such a high and intrinsic to the system dynamic power consumption, we propose to modulate the clock gates of an IP block. The significant size LC reported in previous publications [10], [12] is thus removed. The architecture of the WGC is unmodified and generates a watermark sequence, $W M A R K$. The original clock gate control signal, CLK_CTRL, and WMARK further control the enable signal of a clock gate of an IP block, as shown in Fig. 1(b). Analogically, the original clock signal, $C L K$, to the IP block is modified and replaced with the modulated clock signal, $C L K \_W M A R K$. When WMARK is ' 1 ', the clock gate enable is ' 1 ' and $C L K$ is propagated, hence $C L K \_W M A R K=C L K$. When $W M A R K$ is ' ${ }^{\prime}, C L K$ is stopped at the clock gate and $C L K_{-} W M A R K=0$. In case the watermark circuit is active during processor execution, the entire IP block generates significant dynamic power in clock cycles when $W M A R K$ is ' 1 '. However, this may require an additional synchronization between the watermark modulated and other IP blocks, to ensure data is not corrupted, or increase the throughput. Moreover, the size of the IP module must be significant to generate strong enough watermark power signal, due to the background noise produced by the rest of the system. In case the watermark circuit is active while the entire system is inactive, the watermark power is consumed entirely by clock tree buffers. As can be seen in Fig. 2, the clock modulation technique produces higher switching activity in comparison to watermark architecture implementing a load circuit of Fig. 1(a). This means that clock buffers switch twice in a single clock cycle during the rising and falling edges of a clock signal. Therefore, the dynamic power consumed in a single register by clock tree buffers is higher than the dynamic power consumed by data switching in the same register, as shown in Section V. In Section IV, experiments on silicon test chips are performed, to validate if watermark power generated in such way produces high enough amplitude to be detected with Correlation Power Analysis.

\section{WATERMARK DETECTION}

Simulation results in Fig. 3 demonstrate the effect of an additional watermark circuit on the device total power (in relative terms). The watermark power signal (middle) is added to the power consumed by the embedded system (top), and generates the device total power (bottom). Since the watermark power signal is a much lower amplitude, it is deeply embedded in the overall device power signal. We therefore require an analytical technique which determines the possibility and the accuracy of watermark existence. Such a technique is Correlation Power Analysis (CPA) [10], [11]. In this paper, the CPA detection methodology proposed by Becker et al [10] is followed. The CPA technique requires information extracted from the measured power consumption of a device, recorded using an oscilloscope. The relationship between the expected (watermark sequence) and consumed power signals is measured, as shown in (1).

$$
\rho=\frac{N \sum_{i=1}^{N} X_{i} Y_{i}-\sum_{i=1}^{N} X_{i} \sum_{i=1}^{N} Y_{i}}{\sqrt{N \sum_{i=1}^{N} X_{i}^{2}-\left(\sum_{i=1}^{N} X_{i}\right)^{2}} \sqrt{N \sum_{i=1}^{N} Y_{i}^{2}-\left(\sum_{i=1}^{N} Y_{i}\right)^{2}}}
$$

The watermark model vector, $X$, can be represented by a binary sequence. The sampling frequency of an oscilloscope, $f_{s}$, is much greater than the frequency of the system clock, $f_{c l k}$, i.e. $f_{s}>>f_{c l k}$. Hence, the measured signal is represented as vector, $Y$, where each value is the average of power consumed in a single clock cycle [10]. Vectors $X$ and $Y$ represent data obtained over $N$ clock cycles. The result is known as correlation coefficient, $\rho$ [11]. It can vary from -1 to 1 , with 1 representing two identical signals, and -1 representing two identical but inverted signals. When $\rho$ is 0 , no relationship occurs. Since both signals can be out of phase, the watermark vector $X$ is repeatedly rotated by a single clock cycle and $\rho$ is recalculated [10]. The number of rotations equals the period of the watermark sequence. Once all correlation values have been found, they are represented by a spread spectrum graph [10], as shown in Section IV, Fig. 5. The watermark is only regarded as detected if a single significant correlation coefficient can be resolved, as shown in Section IV, Fig. 5(a).

\section{EXPERIMENTAL RESUlts}

To validate the proposed technique discussed in Section II, we fabricated two ASIC designs in TSMC $65 \mathrm{~nm}$ low leakage CMOS technology, with nominal operating voltage of $1.2 \mathrm{~V}$. The designs were completed using industry standard EDA tools. In the first design (chip I), the watermark circuit was embedded as a hard macro block, on a separate power domain. The SoC consists of the ARM Cortex-M0 microprocessor IP core, along with an on-chip bus and numerous commercial IP blocks. In the second design (chip II), the watermark circuit was embedded from RTL description. Therefore, the watermark circuit was propagated through the entire design flow, which is closer to the intended usage scenario when embedding watermarked soft IP. The chip consists of dual core ARM Cortex-A5 microprocessor IP core and caches. The SoC consists of the ARM Cortex-M0 and the watermark circuit. The watermark circuit architecture is the same in both chips, Fig. 4(a). The WGC contains two sequence generators which can be configured as either 32-bit Linear 


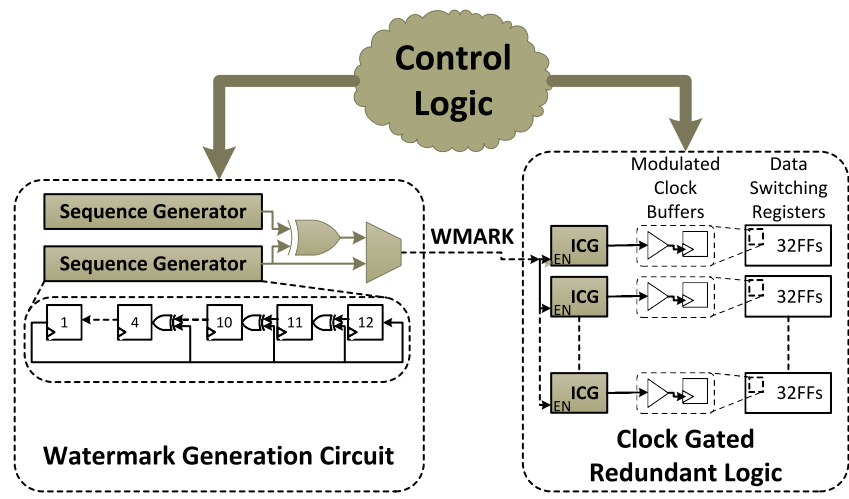

(a)

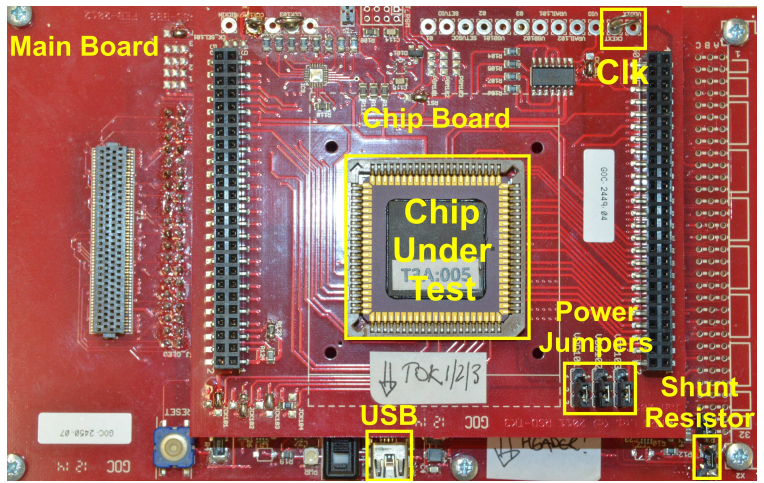

(b)

Fig. 4. (a) Schematic diagram of the clock modulated watermark circuit embedded in test chips. (b) Test board.

Feedback Shift Registers or simple 32-bit circular shift registers. In our experiments, only a single sequence generator was used, configured as 12-bit Linear Feedback Shift Register (LFSR), which generated 12-bit maximum length sequence at $W M A R K$ output signal. The redundant logic circuit contains 1,024 registers, divided into 32 words. The clock signal to each 32-bit word is clock gated using the clock gate cell (ICG). The clock enable signal of each clock gate cell is controlled by $W M A R K$. The clock signal is propagated through all 32 clock gates, when WMARK is ' 1 '. When WMARK is ' 0 ', the clock signal is stopped at ICGs and no dynamic power is consumed. All registers are pre-initialized to ' ${ }^{\prime}$, hence no data switching occurs. It can be seen in Fig. 4(a), that the majority of watermark dynamic power is consumed by clock buffers embedded in registers. Therefore, technique proposed in Section II, can be validated with this experimental setup.

The test board is shown in Fig. 4(b). All power domains were connected using the power jumpers and the total current consumed by the chip was measured, using the shunt $270 \mathrm{~m} \Omega$ resistor. The operating frequency of both chips was $10 \mathrm{MHz}$. The current signal was measured using an Agilent MSO6032A oscilloscope with Agilent 1130A active differential probe, at a sampling frequency of $500 \mathrm{MHz}$. Therefore, 50 samples per single clock cycle were averaged, to find the measured power vector, $Y$. The obtained $N$ clock cycles, for the computation of a single $\rho$, was 300,000 . We attempted to detect the watermark while running the Dhrystone benchmark, which reflects the activities of the integer IP processor core, such as integer arithmetic, string operations, logic decisions and memory accesses in a general computing application [16]. The Dhrystone benchmark was executed on ARM Cortex-M0 on the SoC, on both chips. Although, on chip II Cortex-A5 did not execute any program both cores, along with the on-chip bus were active, which accounted for a significant portion of background noise in the system.

The spectra of correlation results are shown in Fig. 5. Since the period of 12-bit maximum length sequence $\left(2^{12}-1\right)$ is shorter than 300,000 clock cycles, the watermark sequence was repeated multiple times within a vector, $X$. It can be seen, that the correlation peak for chip I occurs at approximately the $3,800^{\text {th }}$ rotation of the watermark sequence, Fig. 5(a), and at approximately the $2,400^{\text {th }}$ rotation of the watermark sequence for chip II, Fig. 5(c). Since no other correlation peaks exist, the watermark can be regarded as detected. To confirm that correlation peaks were not the result of the correlated system noise, the watermark circuit was disabled and experiments were repeated on both chips. As can be seen in Fig. 5(b) and Fig. 5(d), no correlation peaks occurred when watermark power pattern was not present. To investigate the repeatability of detection results, the experiments were performed 100 times on both chips. In Fig. 6 correlation coefficients are shown in a form of a box plot. It can be seen, that medians when $X$ and $Y$ were not in phase is close to 0 . However, when both vectors were in phase medians are much higher and distinctive correlation peak can be distinguished. The variance of all results represented by the box in the figure accounts for $95 \%$ of all correlation coefficients, with extreme values shown as dots. As can be seen, the correlation coefficient peak is present in all experiments on both chips. Therefore, an embedded watermark was successfully detected in all repetitions. The redundant logic in our experiments is a stand-alone circuit, however, in the end application a commercial IP sub-module can be reused with the same results, reducing the area overhead.

\section{Area And Power Overheads}

The area of the current state-of-the-art watermark circuit is greatly occupied by the significant size load circuit (LC), as shown in Section II, Fig. 1(a). In case of system scaling, the size of the watermark generation circuit (WGC) does not change, while the size of the LC varies and increases with the system size. This effect is caused by the Correlation Power Analysis detection technique, which requires substantial watermark power signal to detect an embedded watermark circuit. Various novel detection techniques have been demonstrated in recent publications [17], [18], which detect an embedded circuit of negligible size. However, similarly to many soft IP protection techniques [5]-[9], an access to watermarked design throughout an entire design flow, or access to post-fabricated design internals are necessary for successful detection. This is not possible for many IP vendors. The proposed watermark 


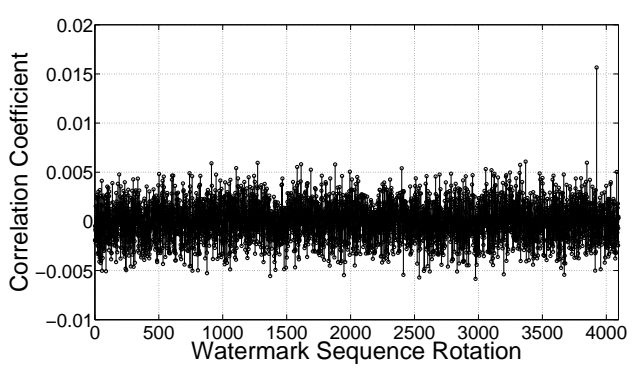

(a)

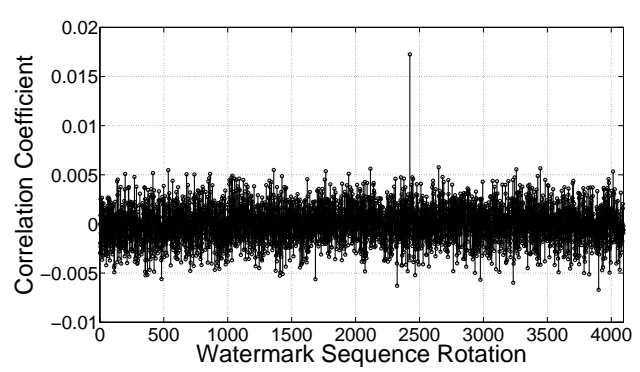

(c)

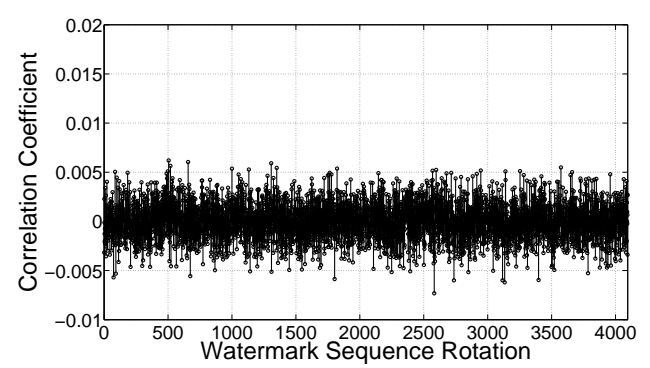

(b)

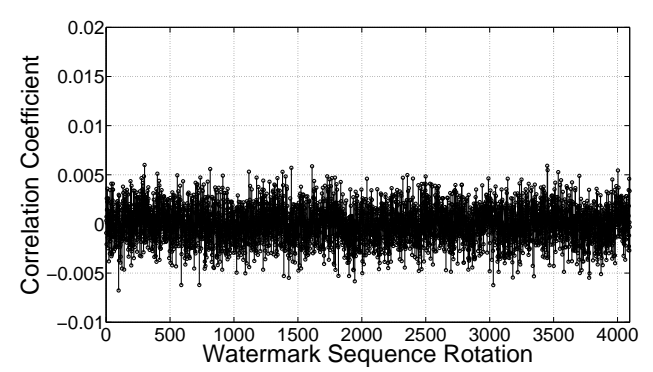

(d)

Fig. 5. Spread spectra of correlation results from test chips. Chip I with (a) active, (b) inactive, watermark circuit. Chip II with (c) active, (d) inactive watermark circuit.

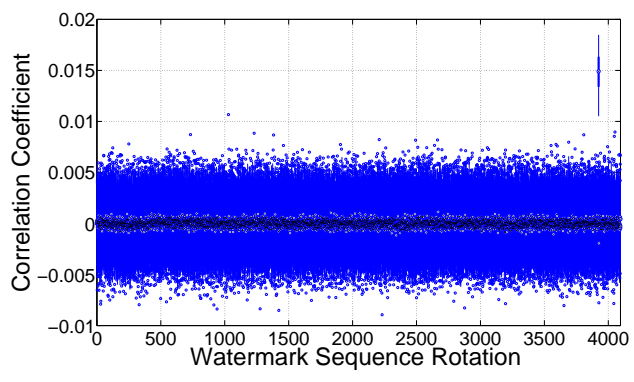

(a)

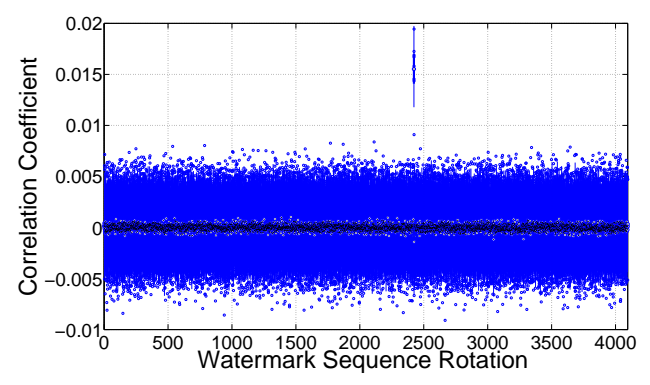

(b)

Fig. 6. Box plots of correlation coefficient results from (a) Chip I and (b) Chip II, when experiment was repeated 100 times.

TABLE I

POWER CONSUmption OF PlACEd AND Routed LoAd CiRCUit

\begin{tabular}{|c|c|c|c|c|}
\hline \multirow{2}{*}{$\begin{array}{c}\text { Load Circuit } \\
\text { Implementation }\end{array}$} & \multicolumn{3}{|c|}{ Power Consumption } & \multirow{2}{*}{$\begin{array}{l}\text { Total Watermark } \\
\text { Dynamic Power }\end{array}$} \\
\hline & Dynamic & Static & Total & \\
\hline $\begin{array}{l}\text { Clock Buffers Modulation } \\
\text { No Data Switching }\end{array}$ & $1.51 \mathrm{~mW}$ & $0.404 \mathrm{uW}$ & $1.51 \mathrm{~mW}$ & $95.6 \%$ \\
\hline $\begin{array}{l}\text { Clock Buffers Modulation } \\
256 \text { Switching Registers }\end{array}$ & $1.80 \mathrm{~mW}$ & $0.407 \mathrm{uW}$ & $1.80 \mathrm{~mW}$ & $96.8 \%$ \\
\hline $\begin{array}{c}\text { Clock Buffers Modulation } \\
512 \text { Switching Registers }\end{array}$ & $2.09 \mathrm{~mW}$ & $0.407 \mathrm{uW}$ & $2.09 \mathrm{~mW}$ & $97.2 \%$ \\
\hline $\begin{array}{l}\text { Clock Buffers Modulation } \\
1024 \text { Switching Registers }\end{array}$ & $2.66 \mathrm{~mW}$ & $0.408 \mathrm{uW}$ & $2.66 \mathrm{~mW}$ & $98 \%$ \\
\hline
\end{tabular}

clock modulation technique enables watermark implementation at RTL, with negligible area overhead, and allows post-fabrication watermark detection with the CPA detection technique. Furthermore, the size of the watermark circuit is the same for all systems, hence does not need scaling, since the watermark architecture only requires implementation of the negligible size WGC.
TABLE II

LOAD CIRCUIT IMPLEMENTATION COSTS

\begin{tabular}{c|c|c}
\hline \hline $\begin{array}{c}\text { Detectable Load } \\
\text { Circuit Dynamic } \\
\text { Power Consumption } \\
P_{L o a d}\end{array}$ & $N=P_{L o a d} /(1.126 u W+1.476 u W)$ & $\begin{array}{c}\text { Number of } \\
\text { Load Circuit Registers } \\
\text { Overhead } \\
\text { Increase }\end{array}$ \\
\hline $0.25 \mathrm{~mW}$ & 96 & $89 \%$ \\
\hline $0.5 \mathrm{~mW}$ & 192 & $94 \%$ \\
\hline $1 \mathrm{~mW}$ & 384 & $97 \%$ \\
\hline $1.5 \mathrm{~mW}$ & 576 & $98 \%$ \\
\hline $5 \mathrm{~mW}$ & 1921 & $99 \%$ \\
\hline $10 \mathrm{~mW}$ & 3843 & $100 \%$ \\
\hline \hline
\end{tabular}

To determine the area overhead reduction of the proposed clock modulation technique to the current state-of-the-art watermark implementation demonstrated in Section II, Fig. 1(a), the power consumption of the fully placed and routed watermark circuit was estimated with Synopsys Primetime$\mathrm{PX}$, using $65 \mathrm{~nm}^{1}$ technology library. The power consumption

\footnotetext{
${ }^{1}$ TSMC $65 \mathrm{~nm}$ low leakage technology library.
} 
of the clock modulated redundant load circuit is shown in Table I. In the top of the table, the LC is implemented as shown in Section IV, Fig. 4(a). Hence, the dynamic power consumption is caused entirely by clock buffers. This implementation was found to account for $95.6 \%$ of total watermark circuit dynamic power. The number of switching registers is further increased, until all 1,024 registers switch when $W M A R K$ is ' 1 '. Therefore, the dynamic power consumption increases and is caused by both data switching and clock buffers modulation. For the comparison, the power consumption of the WGC is the same for all sizes of LC and equals $60 \mu \mathrm{W}$. It can be seen, that the dynamic power consumed by clock buffers is higher than the dynamic power caused by data switching. On average, the dynamic power consumption of a single clock buffer is $1.476 u W$, and data switching in a single register is $1.126 u W$. In Table II, the number of switching registers, $N$, required to implement the LC of Section II, Fig. 1(a), is shown for various system sizes. It is based on the required LC dynamic power consumption (in relative terms), to be easily detected with Correlation Power Analysis. The WGC requires only 12 registers. Therefore, it consumes the same and negligible power, for any system size, and is not included in the table. As can be seen, approximately 580 registers are required to implement the LC with the current state-of-the-art watermark architecture, to consume the same dynamic power as the clock gated redundant circuit in Section IV, Fig. 4(a). With the proposed clock modulation technique, the dynamic power consumed by clock buffers can be obtained through the modulation of clock tree buffers of existing logic, Section II, Fig. 1(b). Hence, an area overhead reduction of $98 \%$ can be achieved, when compared to the technique in [10]. The area overhead reduction depends on the system size, as shown in Table II. As can be seen, the area overhead reduction is less in smaller systems, but still significant when compared to the current state-of-the-art LC watermark implementation. In bigger systems, the area overhead reduction is close to $100 \%$. Moreover, watermark implementation can be system specific. Various top level IP modules or lower level sub-modules can be modulated with the proposed technique. The power overhead of the watermark implementation can be therefore tailored to the system.

\section{IMPROVED RoBUSTNESS}

One of the major cornerstones of all IP watermarking techniques is the robustness against third party removal attacks. It is performed with the aim of removing watermark circuit from the design. In the case of soft IP, a removal attack can be performed at the RTL description level, due to high visibility of the system [2]. Since the current state-of-the-art watermark circuit implements a significant LC, the removal is easily performed. Moreover, as the watermark is a stand-alone circuit, removal has no impact on system performance.

The clock modulation technique proposed in this paper significantly reduces area overhead of the watermark circuit, leading to an enhanced robustness to removal attacks. Since, the watermark generation circuit (WGC) can be embedded in various sub-modules, detection capabilities of an attacker are significantly reduced. As can be seen in Section II, Fig. 1(b), the proposed watermark implementation does not produce a stand-alone circuit, and therefore the system's functionality is greatly impaired when watermark is removed.

\section{CONCLUSIONS}

A novel clock modulation watermark technique for embedded processors has been proposed. The technique was validated with two ASIC designs. The significant area reduction has been demonstrated, when compared to the current stateof-the-art watermark circuit architecture, reported in previous publications, with $98 \%$ area reduction obtained from experimental results. Through embedding the watermark circuit in existing logic, significantly improved robustness to removal attacks was achieved.

The proposed technique offers a considerable area overhead and robustness improvement, when compared to the current state-of-the-art watermark implementation. However, when it is implemented in an embedded processor, the performance is impaired, due to the ' 1 ' and ' 0 ' pattern of a watermark sequence. Moreover, synchronization issues may occur. Addressing these issues form part of our continuing research.

\section{REFERENCES}

[1] G.E. Moore. Cramming More Components onto Integrated Circuits. Electronics, 38(8):114-117, 1965.

[2] VSI Alliance. VSI Alliance Architecture Document: Version 1.0, 1997.

[3] VSI Alliance. Intellectual Property Protection: Schemes, Alternatives and Discussion, August 2001.

[4] R. Torrance and D. James. The State-of-the-Art in IC Reverse Engineering. In CHES. 2009.

[5] A.L. Oliveira. Robust Techniques For Watermarking Sequential Circuit Designs. In DAC, June 1999.

[6] I. Torunoglu and E. Charbon. Watermarking-Based Copyright Protection of Sequential Functions. IEEE Journal of Solid-State Circuits, 35(3):434-440, March 2000.

[7] A. Cui, C. Chang, S. Tahar, and A.T. Abdel-Hamid. A Robust FSM Watermarking Scheme for IP Protection of Sequential Circuit Design. IEEE Transactions on Computer-Aided Design of Integrated Circuits and Systems, 30(5):678-690, May 2011.

[8] A. Abdel-Hamid, S. Tahar, and E.M. Aboulhamid. A Public-Key Watermarking Technique for IP Designs. In DATE, March 2005.

[9] A. Abdel-Hamid and S. Tahar. Fragile IP Watermarking Techniques. In AHS, June 2008.

[10] G. Becker, M. Kasper, A. Moradi, and C. Paar. Side-Channel Based Watermarks for Integrated Circuits. In HOST, June 2010.

[11] E. Brier, C. Clavier, and F. Olivier. Correlation Power Analysis With a Leakage Model. In CHES. 2004.

[12] D. Ziener and J. Teich. FPGA Core Watermarking Based on Power Signature Analysis. In FPT, December 2006.

[13] S. Rusu, S. Tam, H. Muljono, D. Ayers, and J. Chang. A Dual-Core Multi-Threaded Xeon Processor with 16MB L3 Cache. In ISSCC, February 2006.

[14] V. Oklobdzija, V. Stojanovic, D. Markovic, and N. Nedovic. Digital System Clocking: High-Performance and Low-Power Aspects. 2005.

[15] R. Gonzalez and M. Horowitz. Energy Dissipation in General Purpose Microprocessors. IEEE Journal of Solid-State Circuits, 31(9):12771284, September 1996.

[16] R. York. Benchmarking in Context: Dhrystone. ARM, March 2002.

[17] X. Zhang and M. Tehranipoor. RON: An On-Chip Ring Oscillator Network For Hardware Trojan Detection. In DATE, March 2011.

[18] S. Narasimhan, D. Du, R. Chakraborty, S. Paul, F. Wolff, C. Papachristou, K. Roy, and S. Bhunia. Hardware Trojan Detection by MultipleParameter Side-Channel Analysis. IEEE Transactions on Computers, 62(11):2183-2195, August 2012. 